\title{
Corrections
}

\section{Revision der von JOHANN CHRISTIAN FABRICIUS beschriebenen Ichneumonidae (Hymenoptera)}

\author{
KLAUS HORSTMANN
}

On pages 38-39 of the above article (Beitr. Ent. 51 (2001) 1) unfortunately some text has been omitted due to technical error. It should correctly read as follows:

\section{Ichneumon bidentorius FABRICIUS, 1775}

In seiner ersten Beschreibung der Art gibt FABRICIUS (1775: 331) das Propodeum als beiderseits gezähnt an (daher der Name), nach weiteren Beschreibungen (FABRICIUS, 1781: 424; 1793: 145) variiert es zwischen zweigezähnt und ungezähnt. Offensichtlich hat er in der Zwischenzeit neues Material dazubekommen. In Coll. FABRICIUS sind $3 \sigma^{*}$ erhalten, von denen keins mit der Beschreibung von 1775 übereinstimmt. Das erste entspricht der Beschreibung von 1781 (stark beschädigt und unbestimmbar). Das zweite (mit dem Etikett , bidentorius") ist der Abbildung von I. bidentorius durch PANZER (1797: 45, Tab. 15) ähnlich und ist vermutlich deshalb später dazugesteckt worden; es gehört zu Diphyus monitorius (PANZER). Das dritte entspricht keiner der vorliegenden Beschreibungen. Dagegen befindet sich 10 , das mit der Beschreibung von 1775 übereinstimmt, in Coll. BANKS, worauf mich FITTON (in litt.) hingewiesen hat. Meines Erachtens hat FABRICIUS zunächst dieses Exemplar beschrieben, hat aber keinen Sammler und als Fundort nur Europa angegeben, möglicherweise weil seine Notizen unvollständig waren. Später hat er den Typus nicht mehr zur Verfügung gehabt. Die Interpretationen der Art durch andere Autoren folgen meistens der Abbildung durch PANZER, die als o von $D$. quadripunctorius (MÜLLER) identifiziert wird (PEETS, 1912: 73; HORSTMANN, 1982: 240 f.). KRIECHBAUMER (1877: 51 f.) hat die Situation zutreffend diskutiert.

GRAVENHORST (1829: I/427) hat Icbneumon jubilatorius MÜLLER, 1776 und I. citreus CHRIST, 1791 mit I. bidentorius FABRICIUS synonymisiert, bezieht sich aber ausdrücklich auf dessen Beschreibung von 1793. Die Beschreibung von I. jubilatorius durch MÜLLER (1776: 151) stimmt hinreichend gut mit o $\sigma^{*}$ von $D$. quadripunctorius überein. MÜLLER verweist außerdem auf die Abbildung durch SCHAEFFER (1767: Tab. 52, Fig. 5). Diese ist schwer zu interpretieren: Die Färbung der Hinterbeine entspricht $D$. quadripunctorius, die Färbung des Gasters D. mercatorius (FABRICIUS) (siehe auch GRAVENHORST, 1829: I/372). Diese Abbildung wird von PANZER (1804: 70) mit $I$. bidentorius (sensu FABRICIUS 1793: 145) und mit seiner eigenen Abbildung (PANZER, 1797: 45, Tab. 15) identifiziert. Letztere kann ebenfalls als $D$. quadripunctorius gedeutet werden (siehe oben). Die Beschreibung von I. citreus durch CHRIST (1791: 338) stimmt gut mit $o^{\star} \sigma^{\star}$ von $D$. quadripunctorius überein, seine Abbildung (Tab. 33, Fig. 5) weicht in der Zeichnung des Gasters von dieser Art, aber auch von der Beschreibung ab. Aus den genannten Gründen werden I. jubilatorius und $I$. citreus hier als jüngere Synonyme zu $D$. quadripunctorius gestellt (syn. no). 


\section{Ichneumon castigator FABRICIUS, 1793}

GRAVENHORST (1829: I/126) führt Ichneumon certator MÜLLER, 1776, I. cardui SCHRANK, 1786, I. adustus GMELIN, 1790, I. ruficingulus SCHRANK, 1802 und I. pulsator PANZER, 1804 als Synonyme von I. castigator FABRICIUS an, ohne die Priorität zu beachten. An anderer Stelle bringt GRAVENHORST (1829: III/987) noch I. abrogator SCHRANK, 1781 mit I. castigator in Beziehung. DALLA TORRE (1901-1902: 805) stellt deshalb alle diese Taxa mit Bedenken als Synonyme zu I. castigator. Von ihnen entspricht I. abrogator wegen der geringen Körpergröße $(2,5$ lin. = $6 \mathrm{~mm}$; SCHRANK, 1781: 367) keiner der hier diskutierten Arten, wie schon GRAVENHORST (1829: III/987) bemerkt hat. Dieses Taxon wird als unidentifizierte Art zu Icbneumon LINNAEUS (s. l.) gestellt. Die Beschreibung von I. ruficingulus durch SCHRANK (1802: 275) stimmt gut mit o 우 von Ctenichneumon castigator überein, die Synonymisierung beider Taxa wird deshalb bestätigt (siehe oben). I. pulsator ist ein jüngeres Synonym von Coelichneumon bilineatus (GMELIN) (HORSTMANN, 1982: 239).

Die Taxa $I$. certator, $I$. cardui und $I$. adustus werden hier entgegen der Priorität mit dem gültigen Namen Tbyrateles camelinus (WESMAEL, 1845) synonymisiert (syn. n.), mit folgenden Begründungen: Schon FABRICIUS hat $C$. castigator und T. camelinus nicht unterschieden (siehe oben), ebenso wenig GRAVENHORST (TASCHENBERG, 1866: $237 \mathrm{ff}$.). Die Beschreibung von I. cardui (o) durch SCHRANK (1786: 124) stimmt mit T. camelinus gut überein, außerdem wird der Wirt Vanessa cardui (LINNAEUS) (Nymphalidae) für $T$. camelinus häufig genannt. C. castigator parasitiert dagegen an Arten der Noctuidae. I. certator ist sicherlich eine Mischart, aber die erste der von MÜLLER (1776:157) beschriebenen Varietäten stimmt gut mit I. cardui ( $\left.0^{*}\right)$ überein, und SCHRANK (1802: 272) hat beide Taxa ausdrücklich synonymisiert. Die Interpretation von $I$. adustus ist unsicher, weil die Beschreibung durch GMELIN (1790: 2699) sehr kurz ist. Immerhin passt die Beschreibung der Hinterbeine besser zu T. camelinus $\left(\sigma^{*}\right)$ als zu C. castigator $\left(\sigma^{*}\right)$. Da die Bedingungen von Artikel 23.9.1. der Nomenklaturregeln beide erfüllt sind (YU \& HORSTMANN, 1997: 561 und 653), werden hiermit nach Artikel 23.9.2. Amblyteles camelinus WESMAEL, 1845 als Nomen protectum und die Taxa I. certator MÜLLER, 1776, I. cardui SCHRANK, 1786 und I. adustus GMELIN, 1790 als Nomina oblita bezeichnet. 